\title{
Coledococele de pequeño tamaño como causa de dolor epigástrico crónico: diagnóstico y tratamiento mediante CPRE
}

\author{
J. GARCÍA-CANO, J. NIETO', M. A. GODOY², C. J. GÓMEZ RUIZ, N. SÁNCHEZ- \\ MANJAVACAS, A. PÉREZ SOLA
}

Sección de Aparato Digestivo, ${ }^{1}$ Servicio de Medicina Interna, ${ }^{2}$ Servicio de Radiología. Hospital Virgen de la Luz. Cuenca

\begin{abstract}
RESUMEN
El coledococele pertenece al tipo III de los quistes biliares según la clasificación de Alonso-Lej, y son considerados los quistes menos frecuentes entre ellos. La definición habitual del coledococele es la de una dilatación quística de la porción distal intramural del colédoco que protruye en la luz duodenal. La CPRE es una de las pruebas de elección tanto para su diagnóstico como para su tratamiento, especialmente cuando son pequeños y la cavidad quística está habitualmente colapsada. La distensión de la papila ("ballooning") durante la inyección de contraste en la CPRE se considera un signo diagnóstico. Presentamos un paciente con dolor epigástrico crónico a causa de un pequeño coledococele. La Colangiografía por Resonancia Magnética no fue diagnóstica. La CPRE proporcionó el diagnóstico y el tratamiento mediante una esfinterotomía biliar.
\end{abstract}

PALABRAS CLAVE: Quistes biliares. Coledococele. Dolor epigástrico crónico. CPRE.
SMALL SIZE CHOLEDOCHOCELE AS A CAUSE OF CHRONIC EPIGASTRIC PAIN: DIAGNOSIS AND TREATMENT BY MEANS OF ERCP

\begin{abstract}
Choledochocele belongs to type III Alonso-Lej's classification of biliary cysts and they are considered the less frequent of such cysts. The definition most often given of choledochocele is a cystic dilation of the distal intramural portion of the bile duct, protruding into the duodenal lumen. ERCP is one of the diagnostic and therapeutic procedures of choice, specially when they are small and the cystic cavity is usually collapsed. The ballooning of the papilla during contrast injection in ERCP is thought to be a diagnostic sign. We present a patient suffering from chronic epigastric pain due to a small choledochocele. Magnetic Resonance Cholangiopancreatography failed to diagnose it. ERCP offered both diagnosis and treatment by means of biliary sphincterotomy.
\end{abstract}

KEY WORDS: Biliary cysts. Choledochocele. Chronic epigastric pain. ERCP.

García-Cano J, Nieto J, Godoy MA, Gómez Ruiz CJ, Sánchez-Manjavacas N, Pérez Sola A. Coledococele de pequeño tamaño como causa de dolor epigástrico crónico: diagnóstico y tratamiento mediante CPRE. An Med Interna (Madrid) 2005; 22: 591-593.

\section{INTRODUCCIÓN}

La epigastralgia crónica es un problema clínico frecuente. Las causas más habituales son las enfermedades del tracto digestivo superior relacionadas con el ácido y la infección por Helicobacter pylori, así como los problemas bilio-pancreáticos comunes (complicaciones de la patología litásica biliar, pancreatitis). En su evaluación suelen emplearse inicialmente la gastroscopia y la ecografía abdominal transcutánea convencional, además de determinaciones analíticas básicas. Cuando todas estas pruebas no llegan a un diagnóstico definitivo, los enfermos pueden ser diagnosticados de dispepsia no ulcerosa. Si las molestias persis- ten suele recurrise a otras pruebas como la Tomografía Computarizada (TC) o la Resonancia magnética (RM).

Presentamos un caso de epigastralgia crónica, para cuyo diagnóstico fue preciso recurrir a la Colangiopancreatografía retrógrada endoscópica (CPRE).

\section{CASO APORTADO}

Paciente de 57 años con ingresos hospitalarios repetidos por epigastralgia acompañadas de elevaciones mínimas de la amilasa. El paciente estaba diagnosticado de adenoma prostático. Refería dolor

Trabajo aceptado: 11 de julio de 2005

Correspondencia: J. García-Cano. C/ Federico Mayor Zaragoza, 2, 5A, 16002 Cuenca. e-mail: j.garcia-cano@ erra.es 
epigástrico y distensión abdominal durante las comidas que le obligaba a interrumpir la ingesta. En tres ocasiones el paciente acudió a Urgencias. Se observó únicamente una leve elevación de la amilasa (155 UI, normal < 100). Para intentar llegar a un diagnóstico se realizaron una ecografía abdominal trancutánea, gastroscopia, colonoscopia y TC abdominal. Todas las pruebas fueron normales. Finalmente se realizó una colangiopancreatografía por resonancia magnética (CPRM). En ella sólo se observó que el colédoco y el Wirsung desembocaban separados en la pared duodenal. Inicialmente se interpretó como una variante de la normalidad que puede aparecer hasta casi en un $24 \%$ sujetos normales (1). No obstante, ante lo invalidante de los síntomas (el paciente refería epigastralgia casi con todas las comidas) se le propuso realiazar una colangiopancreatografía retrógrada endoscópica (CPRE).

Existía una historia de alergia a contrastes yodados, por lo que se realizó una profilaxis con glucocorticoides. En la imagen endoscópica la papila presentaba un aspecto fláccido. Se canuló profundamente la vía biliar y se introdujo una guía de 0.025 pulgadas para asegurar el acceso al colédoco. La introducción del contraste para la colangiopancreatografía se realizó desde el mismo orificio papilar. Durante la inyección la papila presentó una distensión visible en la imagen endoscópica y que apareció como una estructura quística en la colangiografía (Fig. 1). El Wirsung también se dibujó durante la inyección de contraste pero se vació inmediatamente por lo que no quedó reflejado en las radiografías. Se realizó el diagnóstico de coledococele y se procedió a seccionarlo mediante una esfinerotomía biliar. El paciente no presentó complicaciones de la intervención endoscópica y el dolor durante la ingesta no ha reaparecido tras dos años de seguimiento.

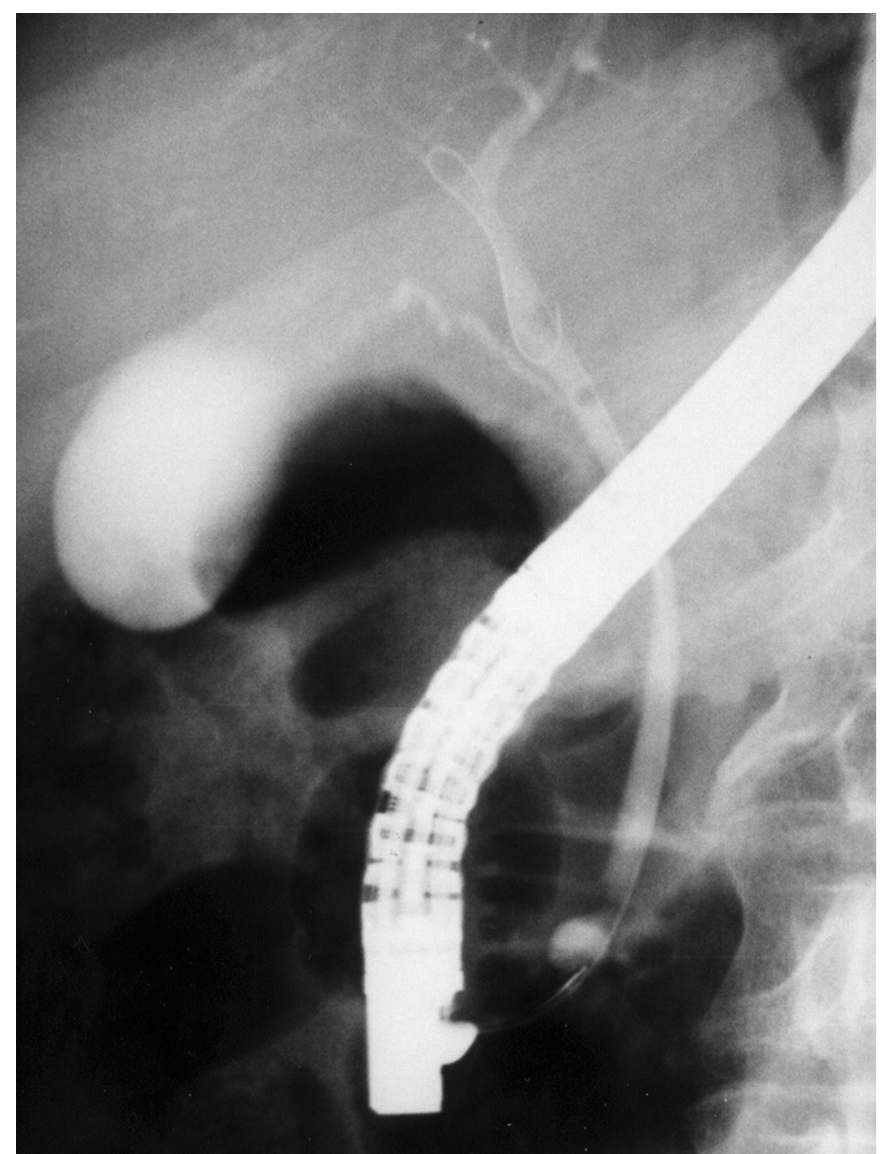

Fig. 1. Colangiografía retrógrada endoscópica. Con la inyección de contraste desde el mismo orificio papilar se ha rellenado la cavidad quística. Es lo que se denomina el signo de la hinchazón o ballooning de la papila. El Wirsung no es visible en la radiografía (aunque se observó en la fluoroscopia) por que el contraste se vació con rapidez.

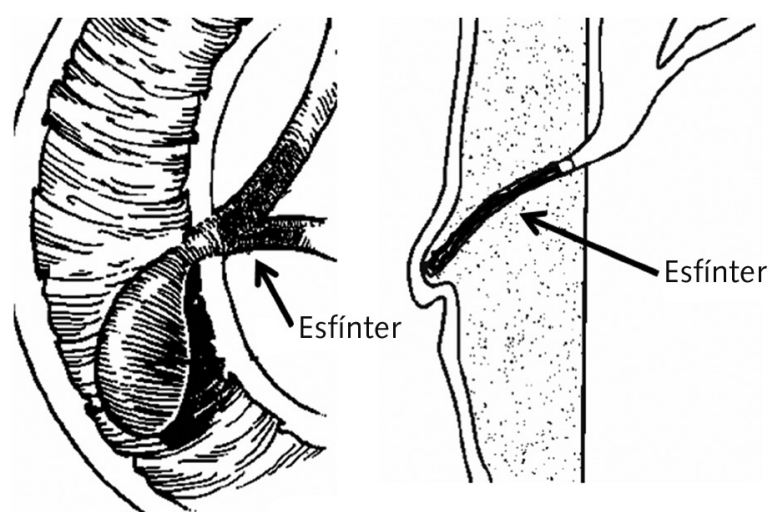

Coledococele

Unión biliopancreática anómala

Fig. 2. Diferencias entre el coledococele y la unión biliopancreática anómala. En esta última patología la bilis y la secreción pancreática se mezclan porque el esfínter de Oddi está sólo en el conducto común. En el coledococele existen los esfínteres biliar y pancreático, aunque ambas secreciones se juntan en la cavidad quística.

\section{DISCUSIÓN}

Los quistes biliares congénitos se encuentran en el 0,32\% de las CPRE (1). Entre ellos, el más raro es el coledococele (tipo III de la clasificación de Alonso-Lej) (2).

El coledococele se suele definir como una dilatación quística de la porción distal intramural del colédoco que protruye en la luz duodenal (Fig. 2).

El aumento de la incidencia en el diagnóstico del coledococele se debe fundamentalmente a la mayor utilización de la CPRE (3), que es especialmente útil para diagnosticar quistes pequeños, cuya cavidad está habitualmente colapsada. Kim y cols. (4) han descrito como signo diagnóstico del coledococele la hinchazón "ballooning" de la papila al inyectar el contraste durante la CPRE. La técnica endoscópica y radiológica debe ser cuidadosa para inyectar el contraste desde la misma papila. Es muy útil insertar en el colédoco una guía para asegurar que el catéter de inyección de contraste esté bien situado en el orificio papilar sin riesgo de que se salga al duodeno.

Los coledococeles pueden subdividirse en cuatro tipos (5): A1, el colédoco y el Wirsung desembocan a través de un solo orificio en el quiste (es el reflejado en la figura 2); A2, colédoco y Wirsung desembocan separados en el quiste; A3, igual que el A2 pero con un quiste sobre todo intramural, que protruye mínimamente el duodeno y que está habitualmente colapsado. Por último, en el subtipo B de coledococele, la papila tiene su orificio normal y el quiste es muy protruyente y sólo comunica con el colédoco.

El caso que describimos corresponde a un A3. Es lógico que al ser muy pequeño y estar colapsado habitualmente, la CPRM no fuera capaz de diagnosticarlo. Por el contrario, la CPRM diagnostica con gran fiabilidad el resto de los quistes biliares, así como las anomalías de la unión biliopancreática (AUBP) (6) (Fig. 2).

Las AUBP son más frecuentes que los quistes biliares $(4,1 \%)(1)$ y se han relacionado con patología coledociana y pancreática. De hecho, en un estudio (1), de 30 pacientes con AUBP, 17 tenían asociado algún tipo de quiste biliar distinto al coledococele. Una de las hipótesis patogénicas es el reflujo mutuo entre las secreciones de ambos conductos, 
debido a la ausencia de mecanismo esfinteriano en su unión (Fig. 2).

Aunque en el coledococele ambos conductos mantienen sus esfínteres, hay también una mezcla de las secreciones en la cavidad quística, por lo que se ha postulado una relación entre coledococele y patología biliopancreática (7). El posible reflujo biliar al páncreas podría explicar el dolor epigástrico que pueden presentar estos pacientes.

Otro aspecto que cabe resaltar en el caso que describimos es el valor diagnóstico de la CPRE, ya que no todas las imágenes obtenidas por CPRM se corresponden con las de la colangiografía endoscópica. Aún asumiendo el riesgo que tiene la CPRE diagnóstica, especialmente de pancreatitis (8), es preciso recurrir a ella en ciertas ocasiones.

En el caso que comentamos, la CPRE fue diagnóstica y terapéutica, ya que la sección del coledococele mediante esfinterotomía biliar solucionó el dolor crónico que padecía el paciente, como se ha descrito en otros casos (4).

\section{Bibliografía}

1. Kim HJ, Kim MH, Lee S-K, Seo D-W, Kim Y-T, Lee D-K, et al. Normal structure, variations, and anomalies of the pancreatobiliary ducts of Koreans: a nationwide cooperative prospective study. Gastrointest Endosc 2002; 55: 889-96.

2. Alonso-Lej F, Rever WB, Pessagno DJ. Congenital choledochal cyst, with a report or 2, and an analysis of 94, cases. Int Abst Surg 1959; 108: 1-30.

3. Park KD, Auh YH, Kim JH, Lee MG, Ha HK, Shim YM, et al. Diagnostic pitfalls in the cholangiographic diagnosis of choledochoceles: cholangiographic quality and its effect on visualization. Abdom Imaging 2001; 26: 48-54.

4. Kim MH, Myung SJ, Lee SK, Yoo BM, Seo DW, Lee MH, et al. Ballooning of the papilla during contrast injection: the semaphore of a choledo- chocele. Gastrointest Endosc 1998; 48: 258-62.

5. Sarris GE, Tsang D. Choledochocele: case report, literature review, and a proposed classification. Surgery 1989: 105: 408-14.

6. Sugiyama M, Baba M, Atomi Y, Hanaoka H, Mizutani Y, Hachiya J. Diagnosis of anomalous pancreatobiliary junction: Value of magnetic resonance cholangiopancreatography. Surgery 1998; 123: 391-7.

7. Kamisawa T, Amemiya K, Nakajima H. Classification of choledochocele and associated pancreatobiliary diseases [Abstract]. Gastrointest Endosc 2003; 57: T1563.

8. García-Cano Lizcano J, González Martín JA, Morillas Ariño MJ, Pérez Sola A. Complicaciones de la Colangiopancreatografía retrógrada endoscópica: estudio en una unidad pequeña de CPRE. Rev Esp Enferm Digest 2004; 96: 163-73. 